\title{
Three-dimensional printing in healthcare
}

\section{Impresión 3D en cuidado de la salud}

\section{A. I. Pérez-Sanpablo ${ }^{1}$, E. Romero-Ávila ${ }^{2}$, A. González-Mendoza ${ }^{1}$}

IInstituto Nacional de Rehabilitación Luis Guillermo Ibarra Ibarra, Mexico City, Mexico

2Department of Rehabilitation and Prevention Engineering, Institute of Applied Medical Engineering - Helmholtz Institute,

RWTH Aachen University, Aachen, Germany

\begin{abstract}
This work aims to briefly present the cutting edge of 3D printing innovation in healthcare. This technology is used for surgical planning, medical education, bioprinting of tissues, and medical equipment spare parts in fields like pharmacology, prosthetics, surgery, and regenerative medicine. A review of the last decade was made in the search engines of PubMed and Espacenet. Three authors reviewed titles, abstracts, and keywords separately to identify studies appropriate to the topic. After the initial examination, complete texts of identified relevant studies were obtained and classified according to the authors. Results were synthesized in a narrative literature review. The revision showed that 3D printing has become of common use in the healthcare system since it allows medical personnel to implement customized solutions for each patient, thus reducing the probability of a false diagnostic or treatment. Major applications among the advantages and disadvantages of 3D printing in healthcare were presented. Nowadays, the main challenge in 3D printing is the cost of the equipment and its manufacturing. In the future, the challenges in cost could be reduced, but processing requirements and limited materials may still need further work.
\end{abstract}

KEYwORDS: 3D printing; stereolithography; medicine 


\section{RESUMEN}

Este artículo pretende mostrar breve y rápidamente la vanguardia del empleo de la impresión 3D en salud. La impresión 3D se utiliza para planificación quirúrgica, educación médica, bioimpresión de tejidos e impresión de repuestos de equipos médicos en campos como farmacología, prótesis, cirugía, ingeniería de tejidos y medicina regenerativa. Se realizó una revisión de publicaciones en la última década en los motores de búsqueda PubMed y Espacenet. Tres autores examinaron de forma independiente títulos y resúmenes para identificar estudios relevantes. Se obtuvieron los textos completos y se clasificaron de acuerdo con todos los autores. Los resultados se sintetizaron en una revisión narrativa de la literatura. La revisión mostró que la impresión 3D se ha vuelto de uso común en el sistema de atención médica, ya que permite al personal médico implementar soluciones personalizadas para cada paciente, lo que reduce la probabilidad de un diagnóstico o tratamiento falso. Se presentaron las principales aplicaciones, así como ventajas y desventajas de la impresión 3D en salud. Hoy en día, el principal desafío en la impresión 3D es el costo del equipo y su fabricación. En el futuro, los desafíos en costos podrían reducirse, pero los desafíos de procesamiento y materiales requieren mayor desarrollo.

PALABRAS CLAVE: Impresión 3D; estereolitografía; medicina

\section{Corresponding author}

TO: Elisa Romero Avila

INSTITUTION: Institute of Applied Medical

Engineering - Helmholtz Institute

ADDRESS: Pauwelsstraße 20, 52074, Aachen, Germany

E-MAIL: romero@ame.rwth-aachen.de

\section{Received:}

26 August 2020

Accepted:

21 January 2021 


\section{INTRODUCTION}

Three-dimensional (3D) printing started as a revolutionizing technology in different fields of healthcare and, at present, has proven to be a useful technology up to a point to become of common use. Clear examples of this are stated in the development of orthotics devices, prostheses, patient-specific implants, Drug Delivery Systems $(D D D)^{[1]}{ }^{[2]}$, realistic anatomic models for education or surgical use ${ }^{[1]}{ }^{[2]}[3]$; the creation of living cells, drugs, protein molecules, that replicate the tissue anatomy, biology, and physiology ${ }^{[2]}{ }^{[4]}$. It is forecasted that the 3D printing industry will grow up to 8.4 billion dollars by $2025^{[5]}$.

3D printing can be defined as a collection of technologies known as rapid prototyping ${ }^{[1]}$ where the conversion of 3D virtual models (models created through Computer-Aided Design (CAD) or image processing) are converted into physical models without the need for specialized tooling, 3D printing includes several established and experimental manufacturing techniques ${ }^{[1]}$ [3] [5]. It is important to mention that any technique used in 3D printing has its limitations and applications. 3D printing is most commonly known as additive manufacturing $(A M)^{[6]}$. The most common way of work of a 3D printing machine is laying one thin layer of material that bonds with another thin layer of material ${ }^{[3]}$. This review aims to identify the latest work and perspectives of 3D printing in healthcare.

\section{MATERIALS AND METHODS}

Searches were carried out in PubMed (ncbi.nlm.nih. gov) and Espacenet (worldwide.espacenet.com) in January, 2021. The studies were in English, Spanish, and French, published between 2009 and 2020. No limitation by its publication status was made. Any type of study was included (experimental studies, observational studies, reviews). The following terms were used for the search: "Printing, ThreeDimensional", "Stereolithography", "Health Services", "Medicine", "Biomedical Research", and "Biomedical
Engineering". In PubMed, previous terms were used as MeSH terms. Three authors reviewed titles, abstracts, and keywords separately to identify studies appropriate to the topics of prostheses and implants, operative and surgical procedures, musculoskeletal system, tissue engineering, cardiovascular system, education, regenerative medicine, pharmacology, skin, otolaryngology, orthopedics, urology, gastroenterology, medical device regulation and, neurology. After the initial examination, complete texts of identified relevant studies were obtained. Studies were classified according to all authors, and disagreements as to which articles were suitable for each category included in this work were resolved by discussion. Results were arranged in a conventional literature review.

\section{RESULTS AND DISCUSSION}

3D printing applied to the area of healthcare has progressed rapidly in the past years. As a result, 6,402 papers (see Figure 1) and 31 patents (see Figure 2) were identified.

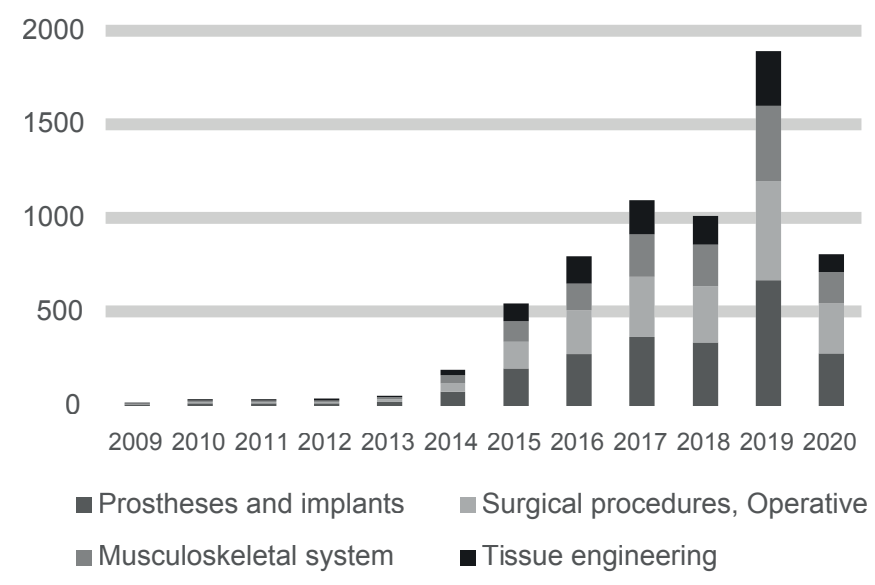

FIGURE 1. The number of publications per year in PubMed about major 3D printing applications in the healthcare field between 2009 and 2020.

Major fields of publication of papers about 3D printing in healthcare include prostheses and implants, surgical procedures, and research about the musculo- 
skeletal system, see Figure 3. Major fields of publication of patents about 3D printing in healthcare include tissue engineering and regenerative medicine, prostheses and implants and, operative and surgical procedures, see Figure 4. In the next sections, a detailed and selective review of major applications among the advantages and disadvantages of 3D printing in healthcare is presented.

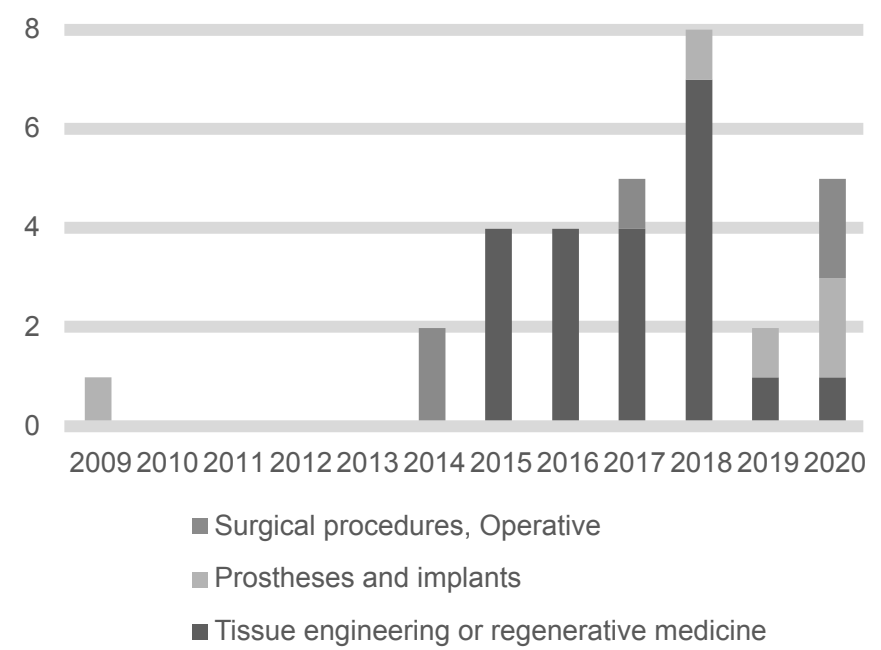

FIGURE 2. The number of patents per year in

Espacenet about major 3D printing applications in the healthcare field between 2009 and 2020.

\section{Advantages of 3D printing}

It can be said that the main advantage of $3 \mathrm{D}$ printing is the personalization of a $3 \mathrm{D}$ model that solves a patient-specific problem at a low-cost burden [6] [7] However, 3D printing technologies also enhance or complement surgical training, research, minimally

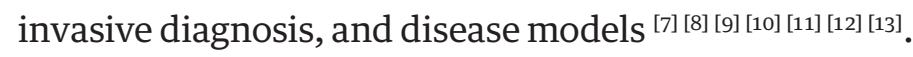

The main advantage of $3 \mathrm{D}$ printing is that it can be used in different applications by only changing the printing technique and material. i.e., in tissue engineering using 3D printing techniques, it is commonly the use of hydrogels because hydrogels allow good permeability of nutrients and facilitate cell growth ${ }^{[14]}$. The use of materials such as metal is used in applications where high mechanical resistance is needed. In applications such as prosthetics or orthotics, materials using metal can be used. In contrast, in applications such as tissue engineering, materials such as bioceramics and bioglass are used since these materials allow the permeability of nutrients ${ }^{[14]}$.
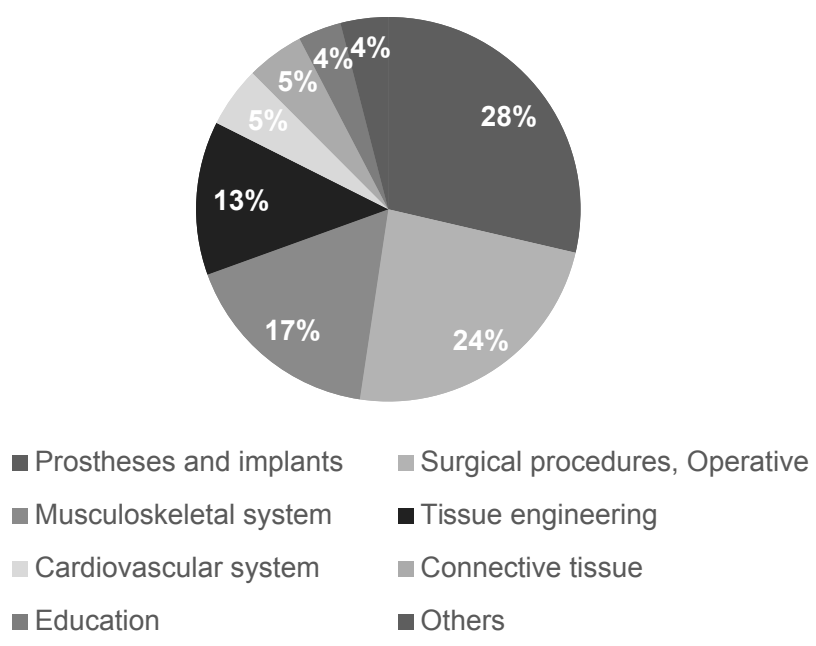

FIGURE 3. Major fields of publication of papers about 3D printing in healthcare are based on the number of publications per year in PubMed between 2009 and 2020.

One of the biggest roadblocks in 3D printing technology is that there is no well-established method of the process that validates the quality check in developing 3-D printed medical devices ${ }^{[8]}{ }^{[15]}$.

The main disadvantage in $3 \mathrm{D}$ printing is found in its manufacturing, where the two challenges are the negative effects of the blemishes situated in the inner part of the 3D model at sub-millimeter scale, which can result from the feed supply that is damaged or machine failures and the effect of the component's placement amid the print on the execution of the material ${ }^{[2]}$.

Regarding the printing technologies, there are still some limitations such as: narrow color range in stereolithography printing (STL), low resolution in fused deposition modeling ( $F D M)$, and poor quality of the surface finish in selective laser melting technique 


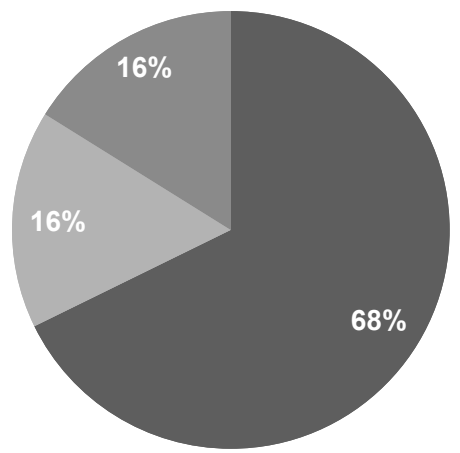

- Tissue engineering or regenerative medicine

Prostheses and implants

n Surgical procedures, Operative

FIGURE 4. Major fields of publication of patents about 3D printing in healthcare are based on the number of publications per year in PubMed between 2009 and 2020.

$(S L M){ }^{[16][17]}$. Although, inkjet bioprinting represents an optimal printing technology in terms of costs and performance, the printed structures may have an irregular and rough shape due to the hydrogels used as bio-inks ${ }^{[16][18]}$. Additionally, the 3D printed material should have specific properties such as flexibility, adaptation, and significant biocompatibility to ensure sufficient vascularization and maturation of the printed tissue ${ }^{[18][19]}$.

\section{D printing in prosthetics and implants}

3D printing in prosthetics can be divided into two types: internal prosthetics and implants and external prosthetics.

Orthoses and splints could be found in external prosthetics. 3D printing has shown various advantages, such as sustainable development and costs reduction. This is particularly relevant in marginalized communities [7] [10] [15] [20]. The most common technique used is additive manufacturing ( $A M)$. The main advantage of 3D printing is efficiency since this model can be modeled using CAD design, allowing to suit the prosthetics perfectly to human anatomy ${ }^{[11]}$.
Internal prostheses and implants are mainly used in orthopedics. Prostheses and implants must mimic the mechanical properties of bone to maximize its performance in the human body ${ }^{[10]}$. The selective laser melting $(S L M)$ technique is a 3D printed technique used to produce internal prostheses and implants. Another technique is selective electron beam melting (SEBM) created by the Swedish company Arcam AB ${ }^{[9]}$. Both processes work by using high energy beams to disintegrate transversal forms into plies of metal powder melding powder particles into a new structure. The major difference between SEBM and SLM techniques is their source of power, the former uses electrons, and the later a laser ${ }^{[9]}$. Enumerated additional requisites of internal prostheses and implants are being biocompatible, allowing cell attachment and expansion, and possess satisfactory porosity [9] [21] [22] [23] [24] [25].

Patents in the field are related to manufacturing devices based on multiple robotic arms and sprayers, manufacturing methods based on the reconstruction of 3D images for facial prosthesis, development of new materials for bone implants, and guiding systems for colocation of dental implants made by stereolithography such

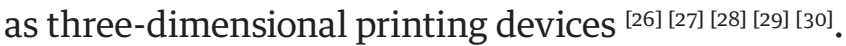

\section{D printing in surgical procedures}

Surgical procedures request high-quality procedural outcomes in combination with optimal safety outcomes ${ }^{[6]}$. 3D printing brought into the surgical field as a tool for simulating all surgical steps ahead of time results in a better comprehension of complex underlying anomalies. This can enhance better diagnostics and assist in surgical planning ${ }^{[6]}{ }^{[7]}$. For example, in craniofacial and maxillofacial surgery where its success has been demonstrated ${ }^{[7]}$. The key components for surgical 3D printing are (1) analysis (critical thinking for problem-solving solutions), (2) planning (endpoint goals are fixed to formulate a surgical plan), (3) virtual surgery (multiple strategies can be run to determine the most optimal approach for surgery), (4) implant 
design and production (biocompatible implants can be printed as an additional complement in surgery), and (5) postoperative analysis (the accuracy of the results can be compared using computed tomography) ${ }^{[6]}$. 3D models regularly take a day or longer to create. This means that 3D models can be used for surgical procedures where long-term planning is involved.

Success stories of application in surgical training include endoscopic ear surgery (the 3D printed models help the surgeons to reduce time), comparison of planning with 3D imaging techniques vs. 3D printing (3D printing allowed surgeons to create better surgical strategies), endoscopic endonasal (where surgeon accelerate their learning process) ${ }^{[6]}$.

Patents in the field are related to methods for fabrication and collocation of $3 \mathrm{D}$ printed materials for facial bone fractures and knee joint replacement [27] [31] [32] [33] 34].

\section{D printing in regenerative medicine}

$3 \mathrm{D}$ printing in regenerative medicine is recognized as an emerging technology that allows the fabrication of biomimetic tissues and organs by cultivating cells on scaffolds. 3D printing in regenerative medicine is made using $C A D$ and computer-aided manufacturing (CAM) methods [18] [35] [36]. Fabricated tissues and organs could be used for medical transplantation in regenerative medicine or drug testing.

To differentiate the specific application of $3 \mathrm{D}$ printing within regenerative medicine, it is commonly referred in the literature as 3D bioprinting. As it can be imagined, 3D printing in regenerative medicine has specific challenges, such as the preservation of biological functions of cells and to mimic the architectures and mechanical properties of biological tissues ${ }^{[19]}$. The $3 \mathrm{D}$ printing technology in the field of regenerative medicine had advanced rapidly since its beginning in 2005, when the first cell printing was performed ${ }^{[36]}$.
Patents in the field are related to the fabrication of encapsulated bodies of cells [37] [38] [39], preparation and application of new materials useful as scaffolds [40] [41] [42] [43] [44] [45] [46] [47] [48] [49] [50] [51] [52] [53][ 54], or new bioinks with improved printability characteristics ${ }^{[55]}$.

\section{Bioprinting, biofabrication and bioassembly}

Bioprinting in regenerative medicine has been used in literature as a general term, which refers to any biofabrication process. However, Moroni et al. have recommended a classification to distinguish each term [56]. Biofabrication is the production by bioprinting or bioassembly of products that are biologically functional using living cells, biomolecules, or biomaterials [56]. Consequently, bioprinting is only one approach to biofabrication. Bioassembly, which is the other major approach of biofabrication, is the process of biofabrication by the assembly of pre-formed cell-containing building blocks ${ }^{[56]}$.

\section{Bioprinting techniques}

The most widely used bioprinting techniques are inkjet-based printing, laser-based printing, extrusion-based printing, and stereolithography STL ${ }^{[18][19]}$.

Inkjet-based printers dispense cells or biomaterials as liquid droplets. The first inkjet-based printer was a modification of a commercial desktop inkjet printer where ink cartridge was substituted by a suspension of cells ${ }^{[19]}$. Inkjet based printers use thermal, piezoelectric, or electromechanical valve mechanisms to print heads to generate the droplets. Thermal printers apply heat to the print head to increase pressure and generate droplets. Piezoelectric printers apply a wave of pressure to split out a droplet of ink. Electromechanical valves printers open and close electromechanical valves to generate droplets. Advantages of inkjet printers are low cost, versatility in managing a wide range of cells and biomaterials with low viscosity ( $<10$ centipoises), high resolution (20-100 micro-meters), high 
speed (1-10,000 droplets per second), precise control of droplets parameters such as volume (1-300 pico-liters), pressure and temperature ${ }^{[19]}$. However, one disadvantage of inkjet-based printers is stress, either thermal o mechanical induced to cells, limitation to use high viscosity cells and biomaterials which could result in obstruction of projecting spouts, the high variability of droplet volume, early condensation, and random dispersion of cells within dispensed volume ${ }^{[19]}$.

Extrusion based printers extrude strands of cells and biomaterials by applying constant pressure through a nozzle by pneumatic or mechanical systems. The advantages of inkjet printers are their versatility to manage a wide range of cells and biomaterials, especially with high viscosity, precise control of nozzle pressure, and nozzle moving speed ${ }^{[19]}$. One disadvantage of extrusion-based printers is the shear stress imposed on cells. The survival rate of cells after extrusion-based printing is $40-86 \%$ lower than inkjet-based printing ${ }^{[19]}$.

Laser-based printers eject droplets of cells and biomaterials from a ribbon structure into a receiving substrate propelled by the high pressure produced by the energy absorption of the laser by the ribbon structure. The ribbon structure is composed of one energy-absorbing layer made of glass coated with nanofilms of gold or titanium and a second layer with a suspension of cells and materials ${ }^{[18]}$. Advantages of laser-based printers are their versatility in managing a wide range of viscosity of cells and biomaterials (1-300 mili-Pascals per second), high density of cell deposition ( $<108$ cells per mili-liter), high resolution (one cell per drop), high speed and visualization of cells during deposition ${ }^{[19]}$.

STL uses a laser of UV light to solidify sections of a photo-sensitive precursor solution layer by layer. The advantages of stereolithography are high resolution (micro-meters). Disadvantages are using only photo-sensitive materials and damage induced to cells and biomaterials for UV exposure ${ }^{[19]}$.

\section{Bio-inks}

Bio-inks are compositions of cells and biomaterials, such as supporting materials and growth factors. Bioinks are mainly composed of hydrogels, polymers with high water content, and cross-links like extracellular matrix. Bio-ink components could be natural such as agarose, alginate, chitosan, collagen, fibrin, gelatin, gel, and gum, or artificial such as hyaluronic acid, pluronic, polyethylene glycol (PEG), and polycaprolactone $(P C L)^{[36]}$. Desirable properties of a bioink are mechanical stiffness, structural stability, biodegradability, thermic stability, biocompatibility and tissue induction and printability ${ }^{[36]}{ }^{[57]}$. The mechanical stiffness of bio-ink should be similar to the mechanical stiffness of healthy tissue, which is important to support loads and maintain the shape of the implant within the body ${ }^{[36]}$. Structural stability and biodegradation denote the maintenance of the structure during printing with a duration of the implant enough to allow tissue remodeling and integration of the cells into the target tissue ${ }^{[36]}$. Thermal stability relates to the property of the bio-ink to remain intact at a relatively high temperature, for example, polymers are solid at $37^{\circ} \mathrm{C}{ }^{[57]}$. Biocompatibility and tissue induction are related to the biological activity and adherence in agreement with cell-matrix interactions of the target

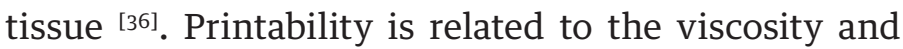
homogeneity of the solution to allow extrusion while maintaining structure after deposition and avoiding high shear stress on cells ${ }^{[36]}$.

\section{D Bioprinting process}

The 3D bioprinting process is like a 3D printing process with some particularities, see Figure 5 . The bioprinting process is initiated by the formation of a tissue or organ blueprint using $C A D$ based on digital images coming from imaging modalities such as Magnetic resonance imaging (MRI) or computed tomography (CT) and the selection of suitable materials according to histological structure, composition, and tissue and organ topology ${ }^{[18]}$. The bioprinting pro- 
cess continues using CAM with the selection of the appropriate printer and bio-ink ${ }^{[18]}$. Finally, the bioprinted tissue or organ is maturated in a bioreactor to mimic in vivo environment of the target tissue ${ }^{[18]}$.

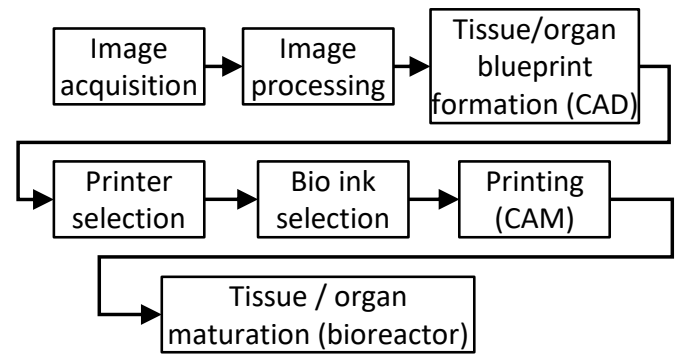

FIGURE 5. 3D bioprinting process.

\section{D printing in research}

According to ${ }^{[5]}$, 3D printing opens new opportunities for scientific research activities. 3D printing allows the creation of phantoms that can help understand physiological processes that are not yet fully comprehended alongside a better understanding of complex pathologies. e.g., hemodynamics can be investigated by velocity-encoded MRI or by optical flow measurements in transparent models.

\section{D printing in medical education and training}

3D printing has proven its value in medical training. As stated in [5] [6] [58], 3D printed models can have a very high value in educational use instead of just images. 3D sections representing a patient with its body structures (bone and vascular) and soft tissues can be printed. To create a 3D model first, an imaging modality is chosen; it can be either through computed tomography or magnetic resonance imaging. From multiple images, volumetric data is acquired. This set of images are put together, and after the noise is smoothed out, a 3D virtual model is created, ready to be printed ${ }^{[2]}{ }^{[3]}$. The accuracy of these models is enough due to the image processing techniques. Within an educational setting, results show that $3 \mathrm{D}$ printing gives a better understanding of anatomy compared to 2D images ${ }^{[6]}$.

\section{D printing in pharmacology}

Patient-specific DDD is the most common technological development in pharmacology ${ }^{[59]}$. At present, the FDA has approved 3D printed $D D D$ in disintegrating dosage forms such as mini-tablets and films that are suitable for children ${ }^{[60]}$.

3D $D D D$ offers favorable circumstances regarding individualized medication conveyance and financial perspective since this is a cheaper alternative to traditional dosage solutions [12] [15] [20] [59] [60] [61]. To print 3D, DDD 5 techniques will be reviewed.

Stereolithography (STL) in pharmacology is the most commonly used technique. Some examples of materials fabricated using this technique are topical patches and microneedles ${ }^{[59]}$. This technique has the disadvantage of short dosage delivery ${ }^{[13]}[59]$.

Selective laser sintering is used to build $D D D$ using polymers such as Nylon, poly-L-lactic acid (PLLA), and $P C L{ }^{[59]}$. This technique gives potential outcomes for controlling the porosity and the medication discharge energy of the $3 \mathrm{D}$ printed structures. The fundamental limitations are the absence of reasonable

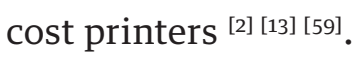

Fused filament fabrication $(F F F)$ or fused deposition modeling (FDM) is the most common technique in the area of pharmacology. The adaptability of FFF permits assembling drug conveyance with different geometries and additionally changed medication discharge profiles for patient-explicit medicines at high reproducibility ${ }^{[13]}[59]$.

A pressure-assisted microsyringe is the newest strategy for $D D D$. Pressure-assisted microsyringe produces $D D D$ from films of poly-lactide-co-glycolide (PLGA) glues, hydrogels, or viscous polymers. However, the amount of medication is limited by the rheological characteristics of the materials ${ }^{[59]}$. 


\section{D printing applications}

\section{D printing applications - Neurologic Applications}

When medical personnel performs neurological surgeries (i.e., Cerebral aneurysm surgery), they can encounter intricate anatomical structures that cannot be seen externally. In order to observe complex anatomical structures, it is commonly used two and three-dimensional images obtained through CT or magnetic resonance imaging MRI ${ }^{[62]}$. Although images allow complex anatomical structures to be visualized, they do not allow medical personnel to appreciate these structures physically. By using imaging techniques, 3D printed anatomical forms can be constructed, which allow preoperative planning.

Preoperative planning brings a few advantages, such as reducing surgery time and decreasing the risk of injuring a patient ${ }^{[63]}$. Neurosurgical models can be printed through FDM, STL, selective laser scintigraphy, and photopolymerization technologies [64]. According to ${ }^{[65]}$ Acrylonitrile Butadiene Styrene (ABS), plastic, and white resin are validated materials to be used in surgical training. Current patents in this field cover the methodology of creating the 3D replica of patient anatomy ${ }^{[66]}$.

3D printing technology can also be used for printing instruments and implants that correspond to individual patient anatomy ${ }^{[67]}$. In the case of $3 \mathrm{D}$ printed implants, advanced materials such as stem cells are used. These methods are considered for either creating new nervous tissue or enhancing the innervation of tissue-engineered constructs [57]. These tissues have basic cellular phenotypes, and previous research shows that their functionality lasts for over two weeks after printing ${ }^{[16]}$. Current patents using steam cells relate to using inkjet printers to place viable cells in a three-dimensional architecture ${ }^{[68][69] .}$

\section{D printing applications - Cardiovascular Applications}

Cardiovascular disease is one of the most severe illnesses that endanger human health, therefore, the best treatment must be applied ${ }^{[70]}$. 3D printing offers the possibility of delivering the best diagnostic and cardiovascular treatment since 3D printing has been used for customized individual printing of cardiovascular models, surgery planning, and simulation of intravascular surgery, resulting in improving the success rate of car-

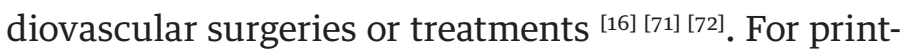
ing 3D cardiovascular models, photopolymer inkjet printers are recommended since they retain a high resolution and can print complex structures ${ }^{[70]}{ }^{[73]}$. The polymeric materials perform the best in terms of quality for cardiovascular models and training tools ${ }^{[70]}$. The current challenge of 3D printing in the cardiovascular field is bio-printing materials used to create functional tissues (stent, valve, tissue-engineered scaffold) that mimic the hearth's physiological function [16] [70] [74] [75] [76]. The biomaterials used for tissue are mainly based on hydrogels since it is a very versatile material. The hydrogel material can adjust its cross-linking density, molecular bonding, and swelling degree to customize its mechanical property ${ }^{[70]}$. The future research of 3D printing cardiovascular disease will focus on bioprinting that can transport blood nutrients ${ }^{[70]}$. Like in 3D printing in neurologic applications, the methodology for creating a 3D printed cardiovascular model is founded in U.S. Pat. No. 20150025666A1 and its application is being applied at the Children National Medical Center. In 2015 the Guangzhou Hongchang registered a technique for printing a small-caliber bioartificial blood vessel that can be used for coronary artery bypass grafting, hemodialysis, and cerebrovascular replacement (C. N. Pat. No. 104771783A).

\section{Musculoskeletal Applications}

Muscles, ligaments, and connective tissues are used on the body to transfer force and facilitate joint movements. Muscles, ligaments, and connective tissue 
might get broken or hurt due to trauma or tumors ${ }^{[77]}$. Muscle, ligaments, and connective tissue treatment refer to tissue transplantation, which its disadvantage is found in the host tissue mechanical properties ${ }^{[77]}$. 3D printing using tissue engineering techniques has the purpose of muscle reconstruction ${ }^{[18]}$. Hydrogels are a common material used in $3 \mathrm{D}$ printing tissue engineering techniques. Hydrogels are designed to act as an artificial extracellular matrix and give living cells an environment to grow ${ }^{[14]}$. Other materials like bioceramics (hydroxyapatite), calcium phosphate, and bioglass have been used for bone regeneration because they are porous, and like hydrogels, facilitate cell growth ${ }^{[14]}$. Common 3D printing techniques for tissue engineering are stereolithography, selective laser sintering, FDM. Previous research has shown that these 3D cell printed muscles exhibit bioelectrical mimetic functionality and structural characteristics ${ }^{[35]}{ }^{[16]}$.

\section{Bones and Cartilage Applications}

The bones are tissues with the ability to self-regenerate and self-repair, but diseases like cancer, infection, trauma, and congenital deformities can prevent bone regenerates or repairs. To treat bone diseases, the transplantation of artificial bone substitutes promotes bone healing by osteogenesis [77]. Bone substitute transplantation treatment has many disadvantages, such as transplant rejection and transmission of diseases. Like in musculoskeletal 3D applications, 3D printing is used in bones and cartilage, tissue engineering using 3D printing techniques, create scaffolds in combinations of cells, materials to improve or replace biological tissues. The $3 \mathrm{D}$ printed scaffolds must use biocompatible materials connected by a porous 3D connected matrix to deliver nutrients ${ }^{[18]}$ [77] ${ }^{[78]}$. A common technique used for creating bone tissue is the hyaluronic acid bio-inks ${ }^{[35]}$. A material that has proven to be useful for $3 \mathrm{D}$ printing bone applications is the polyethylene glycol (PEG) hydrogel, which is mechanically strong, and porous matrixes can be created with this material [35] [57] [77].
The articular cartilage is a smooth surface. The cartilage surface provides support and facilitates joint movements. Since the articular cartilage is avascular and is subjected to external forces, when it degenerates, it is common to see injuries that can evolve in a disease like joint arthritis ${ }^{[14]}$. Tissue engineering using 3D printing techniques, and using hydrogels materials, have the purpose of creating cartilage. Currently, the main problem in 3D printing for articular cartilage is a strong form of the inferior $3 \mathrm{D}$ printed cartilage tissue [77].

\section{Gastroenterology Applications}

Stem cells are also employed in the field of gastroenterology for the $3 \mathrm{D}$ bioprinting of liver tissue, in the form of microstructures that show hepatocyte-like phenotypes and high cell viability [16] [71]. This represents a great advantage in the process of liver transplants since there is currently a limited number of donors. Furthermore, the production of hepatic tissue allows a personalized study about the condition of the liver ${ }^{[71]}$. As seen in other areas, further applications of 3D bioprinting include planning and guidance during surgical operations of the liver for improved screening of the hepatic structures and for medical education, since the $3 \mathrm{D}$ bioprinting of these tissues is a preferable alternative to the use of cadavers in terms of costs and sociocultural issues ${ }^{[79]}$. Patents in this field are related to the methods for $3 \mathrm{D}$ printing of liver tissue ${ }^{\left[{ }^{[0]}\right.}$ and modeling methods for pancreatic surgical planning ${ }^{[45]}$.

\section{Dermatology Applications}

Applications in the field of dermatology include the fabrication of adipose tissue and skin tissue with its equivalents (sweat glands and hair follicles). In this field, the great advantage of the 3D bioprinted tissues is that they are quickly obtained while having enough accuracy as the natural ones ${ }^{[18]}{ }^{[35]}$. Stem cells originating from human adipose tissue are used to create 3D grafts that are complex enough to be compared with the natural adipose tissue. Moreover, the 3D printed 
adipose tissue has the optimal conditions to be used for transplants and allows a personalized study about the condition of the adipose tissue. A great process in the development of skin tissue has been achieved by using collagen matrices embedded with fibroblasts and keratinocytes as bio-inks. This ensures that there is sufficient vasculature to support the tissue since a poor vascularization may have adverse effects on the 3D bioprinted tissue, including necrosis ${ }^{[16]}$. For the reproduction of sweat glands, a combination of components taken from the tissue is used, and its initial experiments have been successful [18] [35]. Patents on this field specify the preparation methods for $3 \mathrm{D}$ printed skin ${ }^{[44][81]}$.

\section{Orthopedic Applications}

The obtention of $3 \mathrm{D}$ printed models to compensate for the loss of bone and cartilage is the main goal within this area by using the inkjet bioprinting technology [18] [82]. With hydrogels as bioinks, the time needed for the creation of the bone tissue is reduced, and its mechanical properties are enhanced. To ensure vascularization, the pores of the printed structures are around $300 \mu \mathrm{m}{ }^{[82]}$. These models are also used in pharmacokinetic studies and for the research of bone-related diseases ${ }^{[82]}$. Several patents are found in this field related to the methods for bone and cartilage printing [26] [31] [34] [43] [47] [50].

\section{Otolaryngology Applications}

One of the clearest examples of the $3 \mathrm{D}$ printing relevance in the field of otorhinolaryngology is the production of hearing aids, as most of the ones available on the market are printed in 3D ${ }^{[83]}$. Patents in this field include the preparation and manufacturing methods of bones with applications in hospitals [53] [69] [84] [85]. Furthermore, as with the above-mentioned applications, 3D printing is also used for surgical education and training since, by providing accurate and complex models, reconstructive surgeries have proven to have improved outcomes and have even been carried out in a reduced period of time ${ }^{[83]}$. The most relevant 3D-printed models/simulators used for surgical education and training in the field of otolaryngology are ${ }^{[17][83]}$ :

- Temporal bones.

- Auricular reconstruction.

- 3D-printed auricular prosthesis.

- Tissue-engineered trachea.

- Ear, sinonasal, skull base, septoplasty, and laryngeal surgery simulators.

\section{Urology Applications}

Applications of 3D-print in urology include education and surgical planning [86] [88]. Printed models include kidney, liver, prostate, ureters, kidney tumors, and renal pelvicalyceal systems ${ }^{[87]}{ }^{[89]}$. The materials used for this go from silicone, wax, or polymers for the kidney models and polyvinyl alcohol hydrogels and 3D-printed injection molds for renal systems. These models are used for renal transplantation and treatment of renal masses with suspected renal cell carcinoma. This is possible since the materials used in $3 \mathrm{D}$ printing share similar characteristics with the organ in terms of shape, elasticity, and mechanical strength ${ }^{[87]}$. Renal pelvicalyceal systems are used in the treatment of nephrolithiasis. Other applications of 3D-printing in urology are the creation of equipment such as stents and trochars ${ }^{[86]}$.

\section{Nutriology Applications}

Within food science, 3D-printing has been proposed as a method to obtain personalized functionalized foods ${ }^{[90]}$. Functional foods (FF) are considered as "foods that supply nutrients and offer potential health benefits that can enhance the well-being of people" ${ }^{\text {[90] }}$. The aim is to offer customized foods that, according to the needs of the user, are supplemented with proteins, sugars, vitamins, and minerals. Examples of ingredients used in 3D-printing are chocolate, pasta, and pork pure, which have been used to produce pizza and enriched cookies ${ }^{[90]}$. 


\section{Radiology Applications}

As well as in other fields, applications of 3D-print in radiology include education and surgical planning [91]. Specifically, within this field, 3D-printing has been used in vascular radiology with the aim to reduce operating times and complications ${ }^{[91]}$. The production of those 3D printed models follows four steps: image acquisition, image segmentation, creation of a $3 \mathrm{D}$ model, and 3D printing ${ }^{[92]}$.

The modality used for the image acquisition in DICOM format (being CT the most common one) depends on the characteristics of the target ${ }^{[93]}$. The segmentation consists of the extraction of regions of interest (ROI) for its 3D reconstruction based on the target tissue and pathophysiology ${ }^{[93]}$. It has been proposed that artificial intelligence can be applied to improve segmentation and also a fusion of multiple imaging modalities ${ }^{[94]}$.

\section{D printing regulations}

In America, the Food and Drug Administration ( $F D A$ ) has emitted, through several centers, guidance documents and regulations to be followed according to the type of application ${ }^{[95]}$ :

- The Center for Devices and Radiological Health $(C R D H)$ supervises medical device applications.

- The Center for Biologics Evaluation and Research controls Biological applications.

- The Center for Drug Evaluation and Research supervises pharmacology applications.

Along with these regulations, the FDA also offers descriptions of the 3D printing processes and learning resources for its application in biomedical sciences. The goal is to accelerate the manufacturing process in $3 \mathrm{D}$ printing and ensure the correct implementation of this technology.

\section{D printing challenges}

The use of 3D printing involves several challenges for its implementation in the healthcare sector. According to a survey made on 700 professional users, the biggest challenges are related to the expenses of the equipment and production, the requirements for its posterior processing, and the short supply of materials ${ }^{[96]}$.

A solution for the cost-related challenges could be the optimization of the manufacturing processes. Another challenge is the resistance to change the guidelines and processes in the healthcare sector ${ }^{[12]}$.

Further challenges are described below. Within the printing process to obtain blueprints of tissues and organs of complex anatomies is a challenge ${ }^{[97]}$. Also, during manufacturing replication of tortuous and thin structures ${ }^{[97]}$, removing negative material from cavities ${ }^{[98]}$ are other challenges. Another specific challenge for regenerative medicine is the integration of tissues and various cell types and the assurance of long-term functionality ${ }^{[4]}$.

Specific challenges for applying 3D printing in pharmacology include regulatory aspects such as assurance of safety and quality of materials and final products ${ }^{[60]}$. Ensuring the proper disposal of printed parts when they are no longer required is an additional regulatory challenge.

These and other challenges need to be addressed to ensure advanced and application of $3 \mathrm{D}$ printing in healthcare.

\section{CONCLUSIONS}

3D printing started as a revolutionizing technology in the different fields of healthcare and, it has demonstrated to be a useful technology up to the point of becoming of common use. This brief review aimed to identify the latest work rapidly and perspectives of 3D printing in healthcare. Major applications among the 
advantages and disadvantages of 3D printing in healthcare were presented. Three-dimensional printing still has challenges that need to be tackled to ensure the progress and application of $3 \mathrm{D}$ printing in healthcare.

\section{AUTHOR CONTRIBUTIONS}

AIPS, ERA and AGM participated in all the stages of the work together, from conceptualization through all the analyses carried and to the writing of all the manuscript versions. 


\section{REFERENCES}

[1] Hurst EJ. 3D Printing in Healthcare: Emerging Applications. J Hosp Librariansh [Internet]. 2016;16(3):255-67. Available from: https://doi.org/10.1080/15323269.2016.1188042

[2] Mukhopadhyay S, Poojary R. A review on 3D printing: Advancement in healthcare technology. In 2018 Advances in Science and Engineering Technology International Conferences (ASET) [Internet]. Abu Dhabi: IEEE; 2018:1-5. Available from: https://doi.org/10.1109/ICASET.2018.8376890

[3] Mishra S. Application of 3D printing in medicine. Indian Heart J [Internet]. 2016;68(1):108-109. Available from: https://doi.org/10.1016/j.ihj.2016.01.009

[4] Ozbolat IT, Peng W, Ozbolat V. Application areas of 3D bioprinting. Drug Discov Today [Internet]. 2016;21(8):1257-1271. Available from: https://doi.org/10.1016/i.drudis.2016.04.006

[5] Chen JKC, Do HTT. Perspective of the 3D Printing Technology Applied on Medical Resource Integration and Service Innovation Business Model. In 2017 Portland International Conference on Management of Engineering and Technology (PICMET) [Internet]. Portland: IEEE; 2017:1-11. Available from: https://doi.org/10.23919/PICIMET.2017.8125298

[6] Langridge B, Momin S, Coumbe B, et al. Systematic Review of the Use of 3-Dimensional Printing in Surgical Teaching and Assessment. J Surg Educ [Internet]. 2018;75(1):209-221. Available from: https://doi.org/10.1016/i.jsurg.2017.06.033

[7] Vaillant T, Steelandt J, Cordonnier A-L, et al. Revue des guides personnalisés à usage unique dans les prothèses totales de genou. Ann Pharm Fr [Internet]. 2018;76(3):228-234. Available from: https://doi.org/10.1016/j.pharma.2017.12.002

[8] Nagarajan NN, Dupret-Bories A, Karabulut E, et al. Enabling personalized implant and controllable biosystem development through 3D printing. Biotechnol Adv [Internet]. 2018;36(2):521533. Available from: https://doi.org/10.1016/j.biotechadv.2018.02.004

[9] Tan XP, Tan YJ, Chow CSL, et al. Metallic powder-bed based 3D printing of cellular scaffolds for orthopaedic implants: A state-ofthe-art review on manufacturing, topological design, mechanical properties and biocompatibility. Mater Sci Eng C Mater Biol Appl [Internet]. 2017;76(1):1328-1343. Available from: https://doi.org/10.1016/j.msec.2017.02.094

[10] Savonen B, Gershenson J, Bow JK, et al. Open-Source ThreeDimensional Printable Infant Clubfoot Brace. JPO J Prosthetics Orthot [Internet]. 2020;32(2):149-158. Available from: https://doi.org/10.1097/JPO.0000000000000257

[11] de Souza MA, Schmitz C, Pinhel MM, et al. Proposal of custom made wrist orthoses based on 3D modelling and 3D printing. In 2017 39th Annual International Conference of the IEEE Engineering in Medicine and Biology Society (EMBC) [Internet]. Jeju: IEEE; 2017:3789-3792. Available from: https://doi.org/10.1109/EMBC.2017.8037682

[12] Awad A, Trenfield SJ, Gaisford S, et al. 3D printed medicines: A new branch of digital healthcare. Int J Pharm [Internet]. 2018;548(1):586-596. Available from: https://doi.org/10.1016/j.ijpharm.2018.07.024
[13] Trenfield SJ, Awad A, Goyanes A, et al. 3D Printing Pharmaceuticals: Drug Development to Frontline Care. Trends Pharmacol Sci [Internet]. 2018;39(5):440-451. Available from: https://doi.org/10.1016/i.tips.2018.02.006

[14] Xia R-Z, Zhai Z-J, Chang Y-Y, Li H-W. Clinical Applications of 3-Dimensional Printing Technology in Hip Joint. Orthop Surg [Internet]. 2019;11(4):533-544. Available from: https://doi.org/10.1111/os.12468

[15] Liang K, Brambilla D, Leroux J-C. Is 3D Printing of Pharmaceuticals a Disruptor or Enabler? Adv Mater [Internet]. 2019;31(5):e1805680. Available from: https://doi.org/10.1002/adma.201805680

[16] Ong CS, Yesantharao P, Huang CY, et al. 3D bioprinting using stem cells. Pediatr Res [Internet]. 2018;83:223-231. Available from: https://doi.org/10.1038/pr.2017.252

[17] VanKoevering KK, Malloy KM. Emerging Role of ThreeDimensional Printing in Simulation in Otolaryngology. Otolaryngol Clin North Am [Internet]. 2017;50(5):947-958. Available from: https://doi.org/10.1016/i.otc.2017.05.006

[18] Huang Y, Zhang X-F, Gao G, et al. 3D bioprinting and the current applications in tissue engineering. Biotechnol J [Internet]. 2017;12(8):1600734. Available from: https://doi.org/10.1002/biot.201600734

[19] Lee VK, Dai G. Printing of Three-Dimensional Tissue Analogs for Regenerative Medicine. Ann Biomed Eng [Internet]. 2017;45:115131. Available from: https://doi.org/10.1007/s10439-016-1613-7

[20] Sandler N, Preis M. Printed Drug-Delivery Systems for Improved Patient Treatment. Trends Pharmacol Sci [Internet]. 2016;37(12):1070-1080. Available from: https://doi.org/10.1016/j.tips.2016.10.002

[21] Shahar FS, Hameed Sultan MT, Lee SH, et al. A review on the orthotics and prosthetics and the potential of kenaf composites as alternative materials for ankle-foot orthosis. J Mech Behav Biomed Mater [Internet]. 2019;99:169-185. Available from: https://doi.org/10.1016/j.jmbbm.2019.07.020

[22] Hooper J, Schwarzkopf R, Fernandez E, et al. Feasibility of singleuse 3D-printed instruments for total knee arthroplasty. Bone Joint J [Internet]. 2019;101-B(7-C):115-120. Available from: https://doi.org/10.1302/0301-620X.101B7.BJJ-2018-1506.R1

[23] Tang X, Qin Y, Xu X, et al. Fabrication and In Vitro Evaluation of 3D Printed Porous Polyetherimide Scaffolds for Bone Tissue Engineering. Biomed Res Int [Internet]. 2019; 2019:2076138. Available from: https://doi.org/10.1155/2019/2076138

[24] Theodoridis K, Aggelidou E, Manthou M, et al. Assessment of cartilage regeneration on 3D collagen-polycaprolactone scaffolds: Evaluation of growth media in static and in perfusion bioreactor dynamic culture. Colloids Surf B Biointerfaces [Internet]. 2019;183(1):110403. Available from: https://doi.org/10.1016/j.colsurfb.2019.110403

[25] Wu Y-HA, Chiu Y-C, Lin Y-H, et al. 3D-Printed Bioactive Calcium Silicate/Poly- $\varepsilon$-Caprolactone Bioscaffolds Modified with Biomimetic Extracellular Matrices for Bone Regeneration. Int J Mol Sci [Internet].2019;20(4):942. Available from: https://doi.org/10.3390/ijms20040942 
[26] Stanislavovna AO, Mironovich BS, Valerevich SS, et al., inventors; Method of producing ceramic sample based on $\beta$-tricalcium phosphate using stereolithography technique for recovering bone tissue [Internet]. RU2729761C1. 2020. Available from: https:// worldwide.espacenet.com/patent/search/family/072086312/ publication/RU2729761C1?q=pn\%3DRU2729761C1

[27] Sergeevich AA, Darchoevich AS, Borisovna AN, et al., inventors; Method of facial prosthesis manufacturing [Internet].

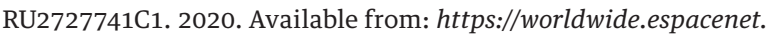
com/patent/search/family/071741362/publication/ RU2727741C1?q=pn\%3DRU2727741C1

[28] Yubo F, Wanru F, Xiaoming L, et al., inventors; Multi-arm cooperation type biological three-dimensional printing device [Internet]. CN110435144A. 2019. Available from: https://worldwide. espacenet.com/patent/search/family/068438558/publication/CN1104 $35144 A$ ? $q=p n \% 3 D C N 110435144 \mathrm{~A}$

[29] Yubo F, Wenyong L, Baosen T, inventors; Intelligent multisource synchronous orienting three-dimensional printing device [Internet]. CN108891025A. 2018. Available from: https://worldwide. espacenet.com/patent/search/family/064344415/publication/CN1088 91025A?q=pn\%3DCN108891025A

[30] Ayberk Y, invertor; Implant guide system [Internet]. WO2009004526A2. 2009. Available from: https://worldwide. espacenet.com/patent/search/family/040019402/publication/WO200 9004526A2?q=pn\%3DWO2009004526A2

[31] Vladimirovich PJ, Akhmadovich SS, Evich SAE, invertors; Method for nail osteosynthesis of facial bone fractures [Internet]. RU2523828C1. 2014. Available from: https://worldwide.espacenet. com/patent/search/family/051265151/publication/ RU2523828C1?q=pn\%3DRU2523828C1

[32] Vladimirovich PJ, Evich SAE, inventors; Method for surgical approach to periapical tissues of jaw [Internet]. RU2523352C1. 2014. Available from: https://worldwide.espacenet.com/patent/ search/family/051217685/publication/ RU2523352C1?q=pn\%3DRU2523352C1

[33] Rui G, Yuyang L, Xiaodong L, et al., inventors; Orbital blow-out fracture titanium mesh prefabrication method [Internet]. CN107374785A. 2018. Available from: https://worldwide.espacenet. com/patent/search/family/060343715/publication/CN107374785A?q= pn\%3DCN107374785A

[34] Sergeevich BS, Andreevich CA, Olegovich da, et al., inventors; Method for replacement of defects of proximal tibia when performing knee joint endoprosthesis replacement and device for its implementation [Internet]. RU2730985C1. 2020. Available from: https://worldwide.espacenet.com/patent/search/family/072238051/ publication/RU2730985C1?q=pn\%3DRU2730985C1

[35] Choi Y-J, Yi H-G, Kim S-W, et al. 3D Cell Printed Tissue Analogues: A New Platform for Theranostics. Theranostics [Internet]. 2017;7(12):3118-3137. Available from: https://doi.org/10.7150/thno.19396

[36] Skeldon G, Lucendo-Villarin B, Shu W. Three-dimensional bioprinting of stem-cell derived tissues for human regenerative medicine. Philos Trans R Soc Lond B Biol Sc i [Internet]. 2018;373(1750): 20170224. Available from: https://doi.org/10.1098/rstb.2017.0224

[37] Yujian K, Xiao Z, inventors; Biobrick used for biological printing and application thereof [Internet]. CN106039419A. 2016. Available from: https://worldwide.espacenet.com/patent/search/family/057484182/ publication/CN106039419A?q=pn\%3DCN106039419A
[38] James KY, Xiao Z, inventors; Compositions for cell-based three dimensional printing [Internet]. WO2016161944A1. 2016. Available from: https://worldwide.espacenet.com/patent/search/ family/057072284/publication/WO2016161944A1?q=pn\%3DWO2016 161944A1

[39] Yujian K, Xiao Z, inventors; Method for preparing constructed body from biobrick containing endothelial cells [Internet].

CN106039409A. 2016. Available from: https://worldwide.espacenet. com/patent/search/family/057484197/publication/CN106039409A?q $=p n \% 3 D C N 106039409 A$

[40] Yuyu H, Shuangshuang M, Wei S, et al., inventors; Frozen gel threedimensional structural body, preparation method and application thereof [Internet]. CN106178110A. 2016. Available from: $h t t p s: / /$ worldwide.espacenet.com/patent/search/family/057458617/ publication $/ C N 106178110 A ? q=p n \% 3 D C N 106178110 A$

[41] Haiqin D, Liumin H, Huiheng L, et al., inventors; Preparation and application of linear biodegradable polyester elastomer with controllable elasticity and shape memory effect [Internet]. CN105504248A. 2016. Available from: https://worldwide.espacenet. com/patent/search/family/055712589/publication/CN105504248A?q =pn\%3DCN105504248A.

[42] Yuxiong G, Pan J, Xiaolong W, et al., inventors; 3D (threedimensional) printing biomedical hydrogel and method for preparing same [Internet]. CN108424533A. 2018. Available from: https://worldwide.espacenet.com/patent/search/family/063164061/ publication $/$ CN108424533A?q=pn\%3DCN108424533A

[43] Zhi H, Lin M, Qing T, et al., inventors; Photo-thermal chemotherapy bone repair material and preparation method of tissue engineering scaffold [Internet]. CN109010925A. 2018. Available from: https:// worldwide.espacenet.com/patent/search/family/064624217/ publication/CN109010925A?q=pn\%3DCN109010925A

[44] Qianqian D, Jianlong J, Aoqun J, et al., inventors; Biological 3D printed full-custom skin and preparation method thereof [Internet]. CN108392676A. 2018. Available from: https://worldwide. espacenet.com/patent/search/family/063101298/publication/CN1083 92676A?q=pn\%3DCN108392676A

[45] Ming'en X, Rui Y, inventors; Pancreatic-like structural body and construction method and application thereof [Internet]. CN111197024A. 2020. Available from: https://worldwide.espacenet. com/patent/search/family/070744047/publication/CN111197024A?q= pn\%3DCN111197024A

[46] Domogatskij, Osidak, inventors; Sterile transparent concentrated solution of biocompatible collagen, method for production and use thereof [Internet]. RU2715715C1. 2020. Available from: https:// worldwide.espacenet.com/patent/search/family/069768223/ publication/RU2715715C1?q=RU2715715C1

[47] Jintao C, Taiying C, Kang L, inventors; 3D printing bone repairing bracket with antibacterial property and preparation method of 3D printing bone repairing bracket [Internet]. CN106729988A. 2017. Available from: https://worldwide.espacenet.com/patent/search/ family/058947966/publication/CN106729988A?q=pn\%3DCN1067299 $88 \mathrm{~A}$

[48] Jun AM, Woo CD, Jin CY, et al., inventors; 3 Wet 3D cell printing using decellularized extracellular matrix [Internet]. KR20180049712A. 2018. Available from: https://worldwide. espacenet.com/patent/search/family/062185559/publication/KR2018 0049712A?q=pn\%3DKR20180049712A 
[49] Peng P, Baoqing Y, Xu Z, et al., inventors; Three-dimensional printing strontium-containing mesoporous bioglass bracket loaded with soy isoflavone and preparation method thereof [Internet]. CN106267375. 2017. Available from: https://worldwide.espacenet. com/patent/search/family/057673419/publication/CN106267375A?q= pn\%3DCN106267375A

[50] Yujiang F, Xuan P, Yaning W, et al., inventors; Bionics design boneline porous bone product and preparation method and purpose thereof [Internet]. CN105877874A. 2016. Available from: https:// worldwide.espacenet.com/patent/search/family/057012628/ publication/CN105877874A?q=pn\%3DCN105877874A

[51] Qi L, Rong W, Weiyi Y, et al., inventors; Three-dimensional biological scaffold, preparation method and applications thereof [Internet]. CN109381739A. 2019. Available from: https://worldwide. espacenet.com/patent/search/family/065412336/publication/CN1093 $81739 A ? q=p n \% 3 D C N 109381739 \mathrm{~A}$

[52] Brown A, Chester D, Daniele M, et al., inventor; Three-dimensional printing of colloidal building blocks for wound healing materials [Internet]. WO2019195681A1. 2019. Available from: https:// worldwide.espacenet.com/patent/search/family/068101458/ publication $/$ WO2019195681A1?q=pn\%3DWO2019195681A1

[53] Wenrui C, Cuihai D, Wenbo H, et al., inventors; Multi-pore bionic skull repair material and individualized manufacturing method [Internet]. CN108273137A. 2018. Available from: https://worldwide. espacenet.com/patent/search/family/062802922/publication/CN1082 $73137 A$ ? $q=p n \% 3 D C N 108273137 \mathrm{~A}$

[54] Chunyan B, Yujie H, Qiuning L, et al., Preparation, raw material, product and application of photo-coupling co-crosslinking hydrogel material [Internet]. CN109776450A. 2019. Available from: https://worldwide.espacenet.com/patent/search/family/066495619/ publication/CN109776450A?q=pn\%3DCN109776450A

[55] Yingfang A, Guocheng D, Xiaoqing H, et al., inventors; Preparation method of cell 3D printing bio-ink with good printability [Internet]. CN111544657A. 2020. Available from: https://worldwide. espacenet.com/patent/search/family/071999658/publication/CN1115 44657A?q=pn\%3DCN111544657A

[56] Moroni L, Boland T, Burdick JA, et al. Biofabrication: A Guide to Technology and Terminology. Trends Biotechnol [Internet]. 2018 36(4):384-402. Available from: https://doi.org/10.1016/j.tibtech.2017.10.015

[57] Aljohani W, Ullah MW, Zhang X, et al. Bioprinting and its applications in tissue engineering and regenerative medicine. Int $\mathrm{J}$ Biol Macromol [Internet]. 2018;107(Part A): 261-275. Available from: https://doi.org/10.1016/j.ijbiomac.2017.08.171

[58] Zhang YS, Oklu R, Dokmeci MR, et al. Three-Dimensional Bioprinting Strategies for Tissue Engineering. Cold Spring Harb Perspect Med [Internet]. 2017;8(2):a025718. Available from: https://doi.org/10.1101/cshperspect.a025718

[59] Palo M, Holländer J, Suominen J, et al. 3D printed drug delivery devices: perspectives and technical challenges. Expert Rev Med Devices [Internet]. 2017;14(9):685-696. Available from: https://doi.org/10.1080/17434440.2017.1363647

[60] Preis M, Oblom H. 3D-Printed Drugs for Children-Are We Ready Yet? AAPS PharmSciTech [Internet]. 2017;18:303-308. Available from: https://doi.org/10.1208/s12249-016-0704-y

[61] Kaae S, Lind JLM, Genina N, et al. Unintended consequences for patients of future personalized pharmacoprinting. Int J Clin Pharm [Internet]. 2018;40:321-324. Available from: https://doi.org/10.1007/s11096-018-0596-x
[62] Randazzo M, Pisapia JM, Singh N, et al. 3D printing in neurosurgery: A systematic review. Surg Neurol Int [Internet]. 2016;7(S33):S801-S809. Available from: https://doi.org/10.4103/2152-7806.194059

[63] Shah KJ, Peterson JC, Chamoun R. 3D Printed Models in Neurosurgical Training BT - Comprehensive Healthcare Simulation: Neurosurgery. In: Alaraj A (ed). Comprehensive Healthcare Simulation: Neurosurgery. Comprehensive Healthcare Simulation [Internet]. Cham: Springer International Publishing; 2018: 47-64. Available from: https://doi.org/10.1007/978-3-319-75583-0_4

[64] Vakharia VN, Vakharia NN, Hill CS. Review of 3-Dimensional Printing on Cranial Neurosurgery Simulation Training. World Neurosurg [Internet]. 2016;88:188-198. Available from: https://doi.org/10.1016/j.wneu.2015.12.031

[65] McMillan A, Kocharyan A, Dekker SE, et al. Comparison of Materials Used for 3D-Printing Temporal Bone Models to Simulate Surgical Dissection. Ann Otol Rhinol Laryngol [Internet]. 2020;129(12):1168-1173. Available from: https://doi.org/10.1177/0003489420918273

[66] Wu A, inventor; Single-action three-dimensional model printing methods [Internet]. US8579620B2. 2011. Available from: $h t t p s: / /$ patents.google.com/patent/US8579620B2/en

[67] Pucci JU, Christophe BR, Sisti JA, et al. Three-dimensional printing: technologies, applications, and limitations in neurosurgery. Biotechnol Adv [Internet]. 2017;35(5):521-529. Available from: https://doi.org/10.1016/j.biotechadv.2017.05.007

[68] Boland T, Wilson JWC, Xu T, inventors; Ink-jet printing of viable cells [Internet]. US7051654B2. 2003. Available from: https:// patents.google.com/patent/US7051654B2/en

[69] Kang H-W, Lee SJ, Atala A, Yoo JJ, inventors; Integrated organ and tissue printing methods, system and apparatus [Internet]. US20120089238A1. 2010. Available from: https://patents.google. com/patent/US20120089238A1/en

[70] Wu Y, Vida VL, Zheng M, Yang J. Progress and Prospects of Cardiovascular 3D Printing BT - Cardiovascular 3D Printing: Techniques and Clinical Application. In: Yang J, Lee AP-W, Vida VL (eds). Cardiovascular 3D Printing [Internet]. Singapore: Springer Singapore; 2021: 179-185. Available from: https://doi.org/10.1007/978-981-15-6957-9_13

[71] Zhang YS, Yue K, Aleman J, et al. 3D Bioprinting for Tissue and Organ Fabrication. Ann Biomed Eng [Internet]. 2017;45:148-163. Available from: https://doi.org/10.1007/s10439-016-1612-8

[72] Ferrari E, Gallo M, Wang C, et al. Three-dimensional printing in adult cardiovascular medicine for surgical and transcatheter procedural planning, teaching and technological innovation. Interact Cardiovasc Thorac Surg [Internet]. 2020;30(2):203-214. Available from: https://doi.org/10.1093/icvts/ivz250

[73] Xu C, Liang J, Yang J. History of Cardiovascular 3D Printing BT Cardiovascular 3D Printing: Techniques and Clinical Application. In: Yang J, Lee AP-W, Vida VL (eds) Cardiovascular 3D Printing [Internet]. Singapore: Springer Singapore; 2021: 1-2. Available from: https://doi.org/10.1007/978-981-15-6957-9_1

[74] Valverde I. Three-dimensional Printed Cardiac Models: Applications in the Field of Medical Education, Cardiovascular Surgery, and Structural Heart Interventions. Rev Esp Cardiol (Engl Ed) [Internet]. 2017;70(4):282-291. Available from: https://doi.org/10.1016/j.rec.2017.01.012 
[75] Baribeau Y, Sharkey A, Mahmood E, et al. Three-Dimensional Printing and Transesophageal Echocardiographic Imaging of Patient-Specific Mitral Valve Models in a Pulsatile Phantom Model. J Cardiothorac Vasc Anesth [Internet]. 2019;33(12):3469-3475. Available from: https://doi.org/10.1053/j.jvca.2019.07.141

[76] Wang Z, Mithieux SM, Weiss AS. Fabrication Techniques for Vascular and Vascularized Tissue Engineering. Adv Healthc Mater [Internet]. 2019;8(19):1900742. Available from: https://doi.org/10.1002/adhm.201900742

[77] Mok S-W, Nizak R, Fu S-C, et al. From the printer: Potential of three-dimensional printing for orthopaedic applications. J Orthop Transl [Internet]. 2016;6:42-49. Available from: https://doi.org/10.1016/j.jot.2016.04.003

[78] Wang Y, Gao M, Wang D, et al. Nanoscale 3D Bioprinting for Osseous Tissue Manufacturing. Int J Nanomedicine [Internet] 2020;15:215-226. Available from: https://doi.org/10.2147/IJN.S172916

[79] Alkhouri N, Zein NN. Three-dimensional printing and pediatric liver disease. Curr Opin Pediatr [Internet]. 2016;28(5):626-630. Available from: https://doi.org/10.1097/MOP.0000000000000395

[80] Wei S, Rui Y, inventors; Construction method and application of in-vitro three-dimensional human liver tissue [Internet]. CN106916781A. 2017. Available from: https://patents.google.com/ patent/CN106916781A/en

[81] Jintao C, Taiying C, Kang L, inventors; Skin repair material with biological activity and method for preparing skin repair material [Internet]. CN106421931A. 2017. Available from: $h t t p s: / / p a t e n t s$. google.com/patent/CN106421931A/zh

[82] Arealis G, Nikolaou VS. Bone printing: new frontiers in the treatment of bone defects. Injury [Internet]. 2015;46(S8):S20-2. Available from: https://doi.org/10.1016/S0020-1383(15)30050-4

[83] Kaye R, Goldstein T, Zeltsman D, et al. Three dimensional printing: A review on the utility within medicine and otolaryngology. Int J Pediatr Otorhinolaryngol [Internet]. 2016;89:145-148. Available from: https://doi.org/10.1016/j.ijporl.2016.08.007

[84] Qingzi B, Yunqi B, Jingsong L, et al., inventors; 4D-printing shapememory-polymer-composite-material tracheal stent and preparing method thereof [Internet]. CN108969165A. 2018. Available from: https://patents.google.com/patent/CN108969165A/ en?oq $=C N 108969165 \mathrm{~A}$

[85] Valerevich MS, Aleksandrovich PA, inventors; Method for manufacture of individual implant for skull bone defects replacement [Internet]. RU2644275C1. 2018. Available from: https://yandex.ru/patents/doc/RU2414870C1_20110327

[86] Chen MY, Skewes J, Desselle M, et al. Current applications of threedimensional printing in urology. BJU Int [Internet]. 2020;125(1):1727. Available from: https://doi.org/10.1111/bju.14928

[87] Parikh N, Sharma P. Three-Dimensional Printing in Urology: History, Current Applications, and Future Directions. Urology [Internet]. 2018;121:3-10. Available from: https://doi.org/10.1016/i.urology.2018.08.004
[88] Childs BS, Manganiello MD, Korets R. Novel Education and Simulation Tools in Urologic Training. Curr Urol Rep [Internet]. 2019;20:81. Available from: https://doi.org/10.1007/s11934-019-0947-8

[89] Tatar İ, Huri E, Selçuk İ, et al. Review of the effect of 3D medical printing and virtual reality on urology training with 'MedTRain3DModsim' Erasmus + European Union Project. Turkish J Med Sci [Internet]. 2019;49:1257-1270. Available from: https://doi.org/10.3906/sag-1905-73

[90] Severini C, Derossi A. Could the 3D Printing Technology be a Useful Strategy to Obtain Customized Nutrition? J Clin Gastroenterol [Internet]. 2016;50:S175-S178. Available from: https://doi.org/10.1097/MCG.0000000000000705

[91] Chang D, Tummala S, Sotero D, et al. Three-Dimensional Printing for Procedure Rehearsal/Simulation/Planning in Interventional Radiology. Tech Vasc Interv Radiol [Internet]. 2019;22(1):14-20. Available from: https://doi.org/10.1053/j.tvir.2018.10.004

[92] Hodgdon T, Danrad R, Patel MJ, et al. Logistics of Threedimensional Printing: Primer for Radiologists. Acad Radiol [Internet]. 2018;25(1):40-51. Available from: https://doi.org/10.1016/i.acra.2017.08.003

[93] Mitsouras D, Liacouras P, Imanzadeh A, et al. Medical 3D Printing for the Radiologist. Radiographics [Internet]. 2015;35(7):19651988. Available from: https://doi.org/10.1148/rg.2015140320

[94] Martín Noguerol T, Paulano-Godino F, Martín-Valdivia MT, et al. Strengths, Weaknesses, Opportunities, and Threats Analysis of Artificial Intelligence and Machine Learning Applications in Radiology. J Am Coll Radiol [Internet]. 2019;16(9):1239-1247. Available from: https://doi.org/10.1016/i.jacr.2019.05.047

[95] U.S. Food \& Drug Administration. 3D Printing of Medical Devices. U.S. Food \& Drug Administration [Internet]. 2019. Available from: https://www.fda.gov/medical-devices/products-and-medicalprocedures/3d-printing-medical-devices

[96] Stratasys Direct Manufacturing. Top Challenges to Widespread 3D Printing Adoption. MachineDesing [Internet]. 2019. Available from: https://www.machinedesign.com/3d-printing-cad/ article/21834436/top-challenges-to-widespread-3d-printingadoption

[97] Abudayyeh I, Gordon B, Ansari MM, et al. A practical guide to cardiovascular 3D printing in clinical practice: Overview and examples. J Interv Cardiol [Internet]. 2018;31(3):375-383. Available from: https://doi.org/10.1111/joic.12446

[98] VanKoevering KK, Hollister SJ, Green GE. Advances in 3-Dimensional Printing in Otolaryngology: A Review. JAMA Otolaryngol Head Neck Surg [Internet]. 2017;143(2):178-183. Available from: https://doi.org/10.1001/jamaoto.2016.3002 